\title{
PULSATIONS OF WHITE DWARF STARS WITH THICK HYDROGEN OR HELIUM SURFACE LAYERS
}

\author{
Arthur N. Cox \\ Sumner G. Starrfield \\ Russell B. Kidman \\ W. Dean Pesnell \\ Theoretical Division, MS B288 \\ Los Alamos National Laboratory \\ Los Alamos, New Mexico 87544
}

\section{Introduction}

In order to see if there could be agreement between results of stellar evolution theory and those of nonradial pulsation theory, calculations of white dwarf models have been made for hydrogen surface masses of $10^{-4} M_{\odot}$. Earlier results by Winget et al. (1982) indicated that surface masses greater than $10^{-8} M_{\odot}$ would not allow nonradial pulsations, even though all the driving and damping is in surface layers only $10^{-12}$ of the mass thick. We show that the surface mass of hydrogen in the pulsating white dwarfs (ZZ Ceti variables) can be any value as long as it is thick enough to contain the surface convection zone.

Evolution calculations that produce white dwarfs from the asymptotic giant branch have for a long time found that there seems always to be a small residual hydrogen surface layer. Fujimoto $(1977,1982)$ first pointed out that it was very difficult to remove all the hydrogen from these red stars, because as soon as the hydrogen is almost gone, any reasonable wind would be very weak for the then small radius, high gravity star. This was also the result also of the early calculations of Schönberner $(1979,1981)$, and subsequent ones by Iben and his many collaborators such as Iben and McDonald $(1985,1986)$.

This residual hydrogen has been a problem for those stars that clearly have no surface hydrogen at all, such as the GW Vir variables and the DB white dwarfs. This situation has been elucidated by Iben (1984) who shows that the final abstraction of all the hydrogen is due to a helium shell flash during some stage after the star has left the asymptotic giant branch. Then the increased luminosity creates a surface convection zone that transports the hydrogen to deep, hot levels where it is all burned. This helium flash also makes the star return again to the red giant region for another wind mass loss episode to blow away the remaining hydrogen with again a possible planetary nebula shell illuminated. 
The results of our white dwarf pulsation calculations are mostly based on a Lagrangian method for getting eigensolutions for low order $\mathrm{g}$ modes. The method is described by Pesnell (1987).

Figures 1 and 2 show the growth rates for pulsation modes at the blue edge of the instability strip for, respectively, $10^{-4}$ and $10^{-8} M_{\odot}$ layers of hydrogen at the surface. One can see that in both cases the growth rates increase with period (with $\mathrm{g}$ mode order) up to the longest unstable period, and they range from $10^{-8}$ to $10^{-1}$ per period. This independence of the pulsation upon hydrogen layer thickness agrees with the observed fact that apparently all the white dwarfs that are in the instability strip are pulsating in one or more nonradial modes. If only the thin layer ones were unstable, then a thick hydrogen shell white dwarf would be stable. There would then be some nonpulsating white dwarfs in the ZZ Ceti instability strip.

\section{Pulsation Mechanisms}

In the process of making these calculations it became apparent that the pulsation driving was frequently at the base of the deep convection zone at temperatures far above those where the hydrogen ionization $\kappa$ and $\gamma$ effects operate. We found that the frozen-in convection assumed in this work acts to periodically block the convection luminosity, and the driving was not at all due to the $\kappa$ and $\gamma$ effects. Adaptation of the convection by a crude model shows that convection blocking in some way is necessary to make the star pulsate. Figures 3 and 4 show this convection blocking driving at the effective temperature of $11,000 \mathrm{~K}$ for our 0.6 $M_{\odot}$ models. In all cases we have needed to assume a rather large convection efficiency to produce white dwarf pulsations at all, as others have also noted.

Figure 3 plots versus zone number and surface mass fraction 5 different structure variables. The FRFT is the fraction of the total luminosity that is being carried by radiation. It is less than $10^{-3}$ in the middle of the zone. The mass zoning is also given to indicate that the Lagrangian mass shells range in mass between $10^{15}$ and $10^{20}$ grams in the convection zone and just below where all the driving is present. The $\Gamma_{3}-1$ dip shown at the surface between zones 500 and 600 is due to the hydrogen ionization, and this low $\Gamma_{3}$ makes the gas very compressible so that at the contraction stage of the pulsation the density is increased considerably. The two logarithmic derivatives of the opacity with, respectively, the density and temperature are key variables in determining if there is radiation blocking during the contraction stages. The density derivative is always positive tending toward increased opacity at this time, but often the temperature derivative is negative allowing for a net decrease in the opacity, and radiation leaking at maximum compression.

Figure 4 gives the pulsation driving and damping for the $g_{7}$ to $g_{10}$ modes for $l=2$ for this model. At zone $\mathbf{4 9 0}$ where the driving is a maximum, the radiation blocking mechanisms are not effective because the gamma has already returned to the completely ionized value, and the opacity derivative with respect 
to temperature is very negative. The pulsation driving is clearly operating at the bottom of the convection zone, because of the convection blocking effect, and not because of radiation blocking.

Figures 5 and 6 , however, show that just $500 \mathrm{~K}$ hotter at $11,500 \mathrm{~K}$, very near the pulsation instability strip blue edge, the usual radiation blocking ( $\kappa$ and $\gamma$ effect) mechanisms are operating at the much cooler zones near zones 522 . These temperatures are near $31,000 \mathrm{~K}$ instead of the $94,000 \mathrm{~K}$ for the previous model.

\section{Other Results}

In addition to these results we also have the following details:

1. Modal selection is caused by the interaction of the eigenvector shape with the composition and the convection luminosity gradients.

2. The theoretical blue edge of the pulsational instability strip is between $11,500 \mathrm{~K}$ and $12,000 \mathrm{~K}$. If the observations really give blue edge temperatures up to $13,000 \mathrm{~K}$, we do not know any mechanism for such hot star pulsations. The Greenstein (1982) blue edge, that we agree with, adopts the Hayes-Latham temperature scale.

3. The longest periods (up to over 1000 seconds) are predicted to occur in the middle of the instability strip as observations indicate. They are not so strongly driven at the blue and red edges, and there only the shorter, more unstable periods are predicted.

4. Radial pulsation modes are still definitely predicted. They must be stabilized by time-dependent convection in a way not yet theoretically known.

5. Helium surface white dwarfs pulsate at $24,000 \mathrm{~K}$ to $27,000 \mathrm{~K}$ surface effective temperature. It is possible that a thin hydrogen layer can cover the helium (DBV) star to have an apparent DA white dwarf pulsate in the DB star instability strip.

\section{REFERENCES}

Fujimoto, M.Y. 1977, Pub. Ast. Soc Japan 29, 331.

Fujimoto M.Y. 1982, Ap. J. 257, 752.

Greenstein, J. L. 1982, Ap. J. 258, 661 .

Iben, I. 1984, Ap. J. 277, 333.

Iben, I. and McDonald, J. 1985, Ap. J. 296, 540.

Iben, I. and McDonald, J. 1986, Ap. J. 301, 164.

Pesnell, W.D. 1987 Ap. J. submitted

Schönberner, D. 1979, Astron. Astrophys. 79, 108.

Schönberner, D. 1981, Astron. Astrophys. 103, 119.

Winget, D.E., Van Horn, H.M., Tassoul, M., Hansen, C.J., Fontaine, G. and Carroll, B.W. 1982, Ap. J. Lett. 252, L65. 


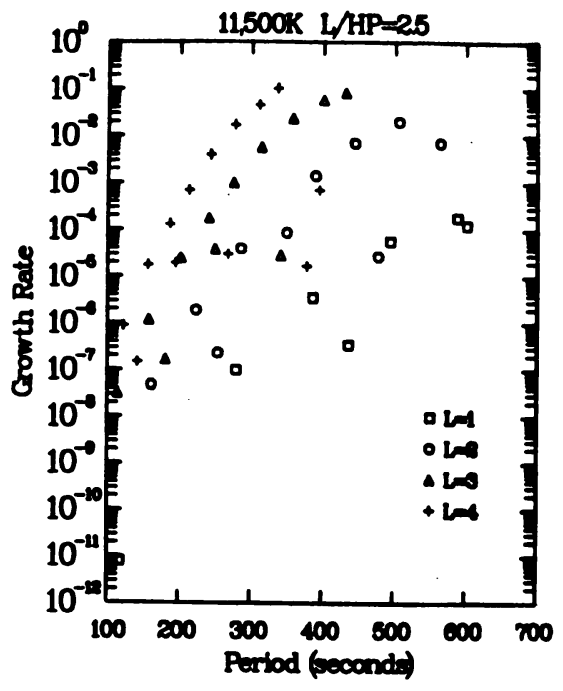

Fig. 1

Growth rate (per period) versus period for $g$ modes in the deep hydrogen model.

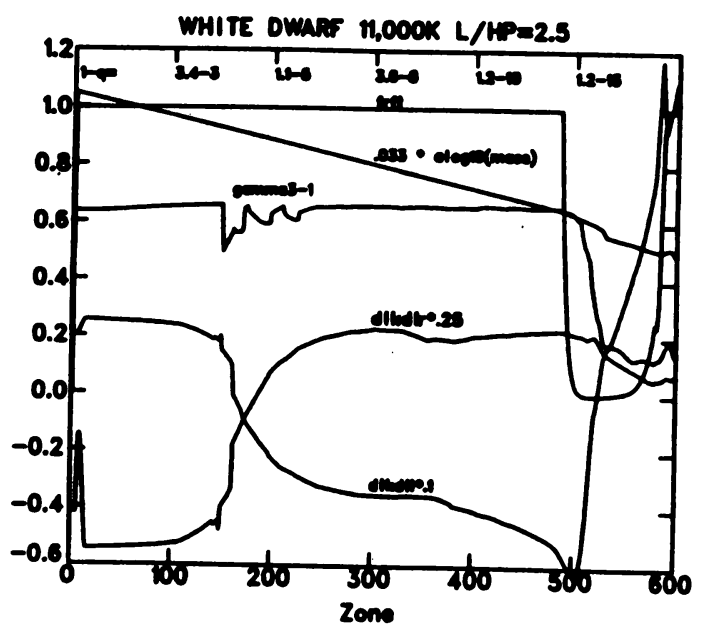

Fig. 3

Structure variables for the $11,000 \mathrm{~K}$ model.

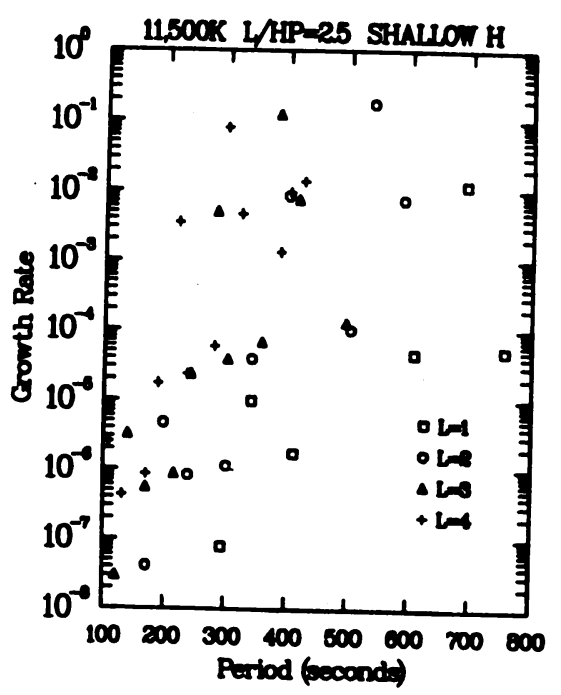

Fig. 2

Growth rate (per period) versus period for $g$ modes in the shallow hydrogen model.

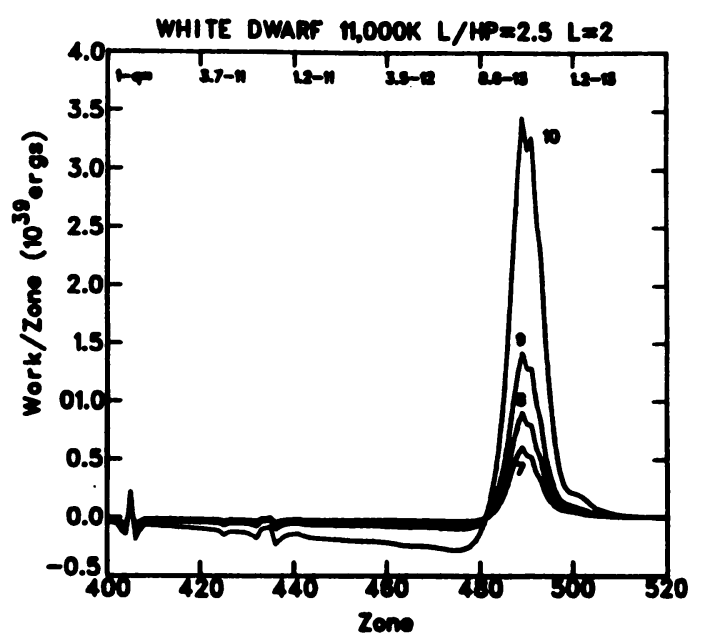

Fig. 4

Work per zone to cause pulsation versus zone number and surface mass fraction for four $g$ modes driven by convection blocking. 


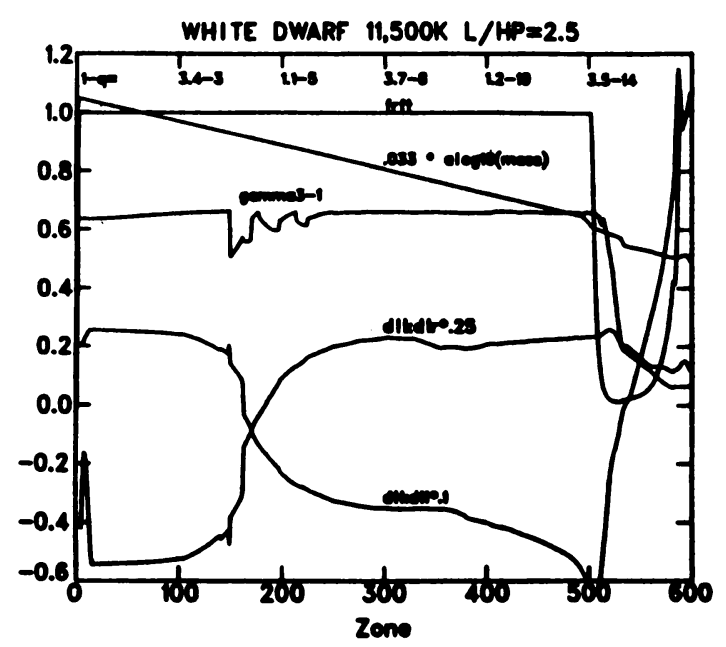

Fig. 5

Structure variables for the 11 , 500K model.

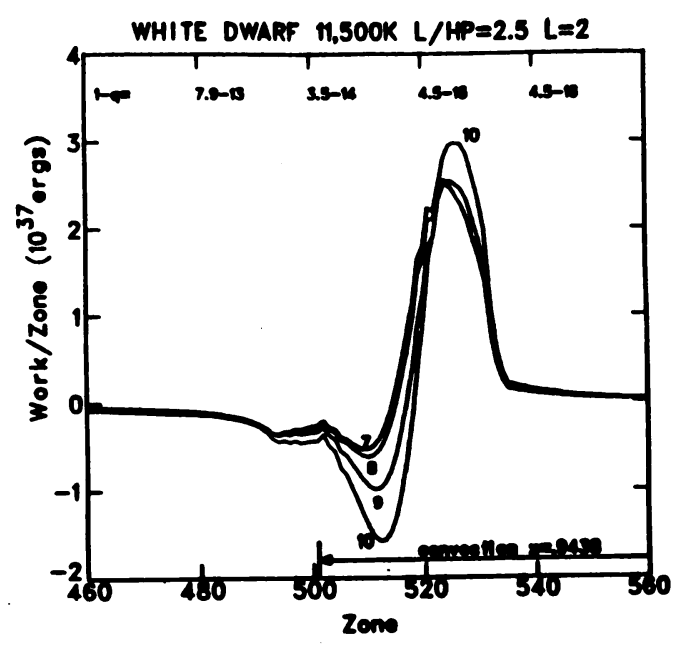

Fig. 6

Work per zones to cause pulsation versus zone number and surface msss fraction for four $g$ modes driven by radiation blocking. 